\title{
A self-reported long-term follow-up of patients operated with either shortening techniques or a TachoSil grafting procedure
}

\author{
Marcus Horstmann*, Matthias Kwol, Bastian Amend, Joerg Hennenlotter and Arnulf Stenzl
}

The aim of this article is to analyse the long-term results of different surgical techniques for correction of penile deviations in Peyronie's disease. Patients who underwent surgery for a penile deviation in Peyronie's disease between 1997 and 2007 were included into this study. Cases were retrospectively analysed by chart review. The current situation was evaluated by a 16 -item standardized questionnaire addressing penile straightness, sensation, length, sexual function and satisfaction. Ninety patients were contacted with a return rate of $75(83 \%)$ evaluable questionnaires. Thirty-two patients were operated by shortening techniques (STs) with either Schroeder-Essed $(n=16)$ or Nesbit $(n=16)$. Forty-three were operated by a plaque in-/excision and defect covering by TachoSil method (TM). Both groups were similar regarding age (ST 56 years, TM 57 years), comorbidities, mean preoperative erection hardness score (EHS; $3.1 \mathrm{ST}, 3.4 \mathrm{TM}$ ) and time of follow-up (total mean, 63 months). Preoperatively ST patients had significantly less plaques $(P<0.05)$ and a lower deviation angle in comparison to TM patients (ST $56^{\circ}$ versus TM $74^{\circ} ; P<0.001$ ). Still TM patients reported slightly better straightening results, but a significantly lower mean EHS (ST 3.3 versus TM 2.6; $P<0.001$ ) during the follow-up. Satisfaction rates were similar in both groups. In conclusion, both techniques revealed to be safe and sustainable successful in penile straightening with better functional outcome of patients operated by STs.

Asian Journal of Andrology (2011) 13, 326-331; doi:10.1038/aja.2010.157; published online 17 January 2011

Keywords: Nesbit, penile deviation; penile reconstruction; Peyronie's disease; Schroeder-Essed; TachoSil grafting

\section{INTRODUCTION}

Peyronie's disease (PD) is an acquired penile condition characterized by fibrous plaques within the tunica albuginea and the subtunical tissue of the corpora cavernosa. ${ }^{1,2}$ Plaques are considered responsible for the main symptoms: pain, penile deviation and erectile dysfunction which can make sexual intercourse difficult or impossible. Overall prevalence is estimated between 3 and $5 \%{ }^{3,4}$ It is known to be highest at the ages of 40-60 years. Even though several risk factors and associations with other diseases are known (Dupuytren's contracture, hyperlipidemia, diabetes, etc.), the exact aetiology of PD remains unclear. ${ }^{5,6}$ Most commonly discussed hypotheses for the development of PD are trauma (microtrauma), inflammatory (autoimmune or infectious) and/or ischemic processes. ${ }^{1,7,8}$ Currently most non-surgical treatment forms like medical or physical applications are symptom based ${ }^{9-12}$ and evidence-based treatment has recently been tried to establish. ${ }^{13}$ Although one-third of the patients will improve clinically during the natural course of this disease, another third will seek surgical correction. ${ }^{14}$ Several surgical techniques have been developed. Most commonly shortening techniques (STs) like Schroeder-Essed and Nesbit are used. ${ }^{15-17}$ Both techniques are based on the principal that the longer (convex) part of the deviated penile shaft is shortened by plication sutures (Schroeder-Essed) or by excisions of tissue parts (Nesbit). Because these techniques are criticized to result in penile shortening, grafting procedures became popular especially in PD with high degrees of deviation. According to these techniques the plaque (located on the shorter (concave) part of the deviation) is either incised or completely removed. The consecutive defect of the corpora cavernosa is then covered by a graft which can consist of different materials: ${ }^{18}$ autologous materials like vena saphena magna, ${ }^{19,20}$ allogenic pericardial ${ }^{21}$ or dermis grafts, ${ }^{22,23}$ but also xenogenous materials like small intestinal submucosa ${ }^{24}$ or collagen matrices ${ }^{25}$ are applied. Even though some publications already report successful surgical treatment, long-term follow-up data especially with regard to the functional outcome remain scarce. ${ }^{14,21,26,27}$

The aim of the present study was to evaluate and compare the selfreported long-term outcome and overall satisfaction in patients treated by either STs or a grafting procedure with TachoSil method (TM) at our institution. (See below for a detailed description of the surgical methods).

\section{PATIENTS AND METHODS}

Study design

The study was approved by the Ethics Committee at the University of Tuebingen (approval number 389/2008BO2). All patients operated between 1997 and 2007 at the University of Tuebingen for penile deviations in PD were included into this retrospective evaluation. 
Clinical pre- and perioperative data were obtained by chart review noting the penile deviation (direction, angle and localisation), pain, erectile function and preceding treatments. General patient information as age, comorbidities, drug use, etc. was added to the database.

To evaluate postoperative outcome, a standardized 16-item questionnaire was developed (Appendix: Questionnaire, translated into English). It included simple questions with predefined answers. To assess erectile function, the erection hardness score (EHS) was applied as one item. ${ }^{28}$ Since the EHS was only developed in $1998,{ }^{29}$ and the study evaluated patients from 1997 to 2007, the preoperative EHS was calculated in patients in whom it was not available by chart review. Charts with conclusive and detailed descriptions of preoperative sexual function including a precise description of erection hardness were available in all patients. Before sending out the questionnaires, patients were informed by telephone calls about the study. Questionnaires were then sent out up to three times to the patients.

\section{Statistical analysis}

All data were collected in a database. The unpaired Student's $t$-test was applied for all comparisons. JMP (SAS Institute Inc., Cary, NC, USA) software was used for all analyses. $P$ values of $<0.05$ were considered significant.

\section{Surgical preparation and techniques}

All interventions were performed under general anaesthesia. Surgery was performed after at least 6 months of stable deviation. Patients counselling included the different techniques and risk factors: postoperative bleeding, penile shortening, loss of sensation, erectile dysfunction and incomplete correction of the deviation. The decision on the surgical technique to be applied was determined individually between patient and surgeon. Preoperative evaluation included physical examination, assessment of erectile function, photo documentation of the erect penis, ultrasound and, if considered necessary, a pharmacologically induced artificial erection.

During the intervention an artificial erection was created first to verify the localisation and angle of the deviation. Then the penis was circumcised unless it has been done before or was explicitly not desired. Afterwards the penis was completely 'degloved' on Buck's fascia and again an artificial erection was created to verify the point of maximal curvature. STs were then performed according to the detailed description of the original surgical techniques previously published. ${ }^{15,16}$

For the grafting procedure with TachoSil (Nycomed GmbH, Konstanz, Germany), 'degloving' of the penis was done similarly to the techniques described above. In all cases with a dorsal deviation a mobilisation of the dorsal neurovascular bundle was performed to fully expose the point of maximal curvature. Then a dorsal incision or a partial and sometimes complete plaque excision was performed. If necessary, several incisions were made. Afterwards, the defect was covered by a TachoSil patch according to the manufacture's descriptions without any sutures. After manual compression, it was re-covered by the neurovascular bundle and a pressure bandage was applied.

\section{Tachosil}

Tachosil is a sterile ready-to-use absorbable sealant patch for topical application that is commercially available in different sizes. The current patch: TachoSil consists of equine collagen that is coated with fibrin glue components, human fibrinogen and human thrombin. It is approved in Europe for supportive haemostatic treatment in surgery.
Its efficacy and safety have been demonstrated in liver resection, ${ }^{30}$ pulmonary resection, ${ }^{31}$ and kidney resection. ${ }^{32}$ Its predecessors TachoComb and TachComb $\mathrm{H}$ have proven to be efficacious haemostats and tissue sealants for several years. Both have also been used in patients of the present study since the evaluated data range from 1997 to 2007 and TachoSil was available on the European market not earlier than 2004. In PD, TachoComb was described as a grafting material by Lahme et al. for the first time in 2002, ${ }^{25}$ and has become widespread in Germany since then. However, to our knowledge, no further experiences with this technique have been published thereafter.

\section{Patients}

After initial telephone contact, questionnaires were sent out to 90 patients operated on PD between 1997 and 2007. Seventy-five patients $(83 \%)$ returned evaluable questionnaires: 32 patients were operated by an ST including 16 Nesbit and 16 Schroeder-Essed patients; 43 patients were operated according to the TM (Table 1). The mean age of patients at the time of surgery and follow-up periods are listed in Table 1.

\section{Preoperative evaluation}

As shown in Table 1, preoperatively the mean deviation angle was $56^{\circ}$ (range, $15-110^{\circ}$ ) in the ST and $74^{\circ}$ (range, $25-110^{\circ}$ ) in the TM group $(P<0.001)$. In both groups the point of maximal deviation was in most of the patients in the middle third (ST: 20 (62\%) versus TM: 24 $(56 \%))$. Most of the patients had a dorsal deviation (ST: 19 (59\%) versus TM 38 (88\%). In $24(75 \%)$ patients of the ST group, plaques were diagnosed and in $42(98 \%)$ patients of the TM group.

Preoperative erectile function was similar in both groups $(P=0.2)$. The average EHS of ST patients was 3.1 (EHS 1: $n=0$; EHS 2: $n=6$; EHS 3: $n=14$; EHS $4: n=12$ ) and of TM patients 3.4 (EHS $1: n=0$; EHS 2: $n=7$; EHS 3: $n=12$; EHS 4: $n=24$ ) (Table 1). Preoperatively, penetration was possible for 13 (41\%) ST and 16 (37\%) TM patients $(P=0.9)$. The incidence of comorbidities was similar in both groups: $10(31 \%)$ ST and 14 (33\%) TM patients were known for hypertonus, 5 (16\%) ST and 8 (19\%) TM patients for diabetes and 4 (12\%) ST and 5 (12\%) TM patients for metabolic syndrome. Six (19\%) ST and 9 (21\%) TM patients suffered from Dupuytren's contracture.

\section{Surgical and perioperative evaluation}

As shown in Table 1, the mean time between diagnosis and surgery was 26 months (range, 9-96) in ST and 22 (range, 6-96) in TM patients $(P=0.4)$ (Table 1$)$. The time of surgery was significantly longer in the TM group. In the ST group, the average operating time was $72 \mathrm{~min}$ (range, 33-115 $\mathrm{min}$ ) and $99 \mathrm{~min}$ (range, 45-177 $\mathrm{min}$ ) in the TM group $(P<0.001)$. In $19(44 \%)$ TM group patients, only an incision of the corpora cavernosa was performed and $24(56 \%)$ had a partial or complete plaque excision.

Table 1 also shows that postoperative haematomas are observed in 6 (19\%) ST and $6(14 \%)$ TM patients. A surgical revision was necessary in 3 patients (2 (6\%) ST patients, 1 (2\%) TM patient). Other postoperative complications were oedema in 6 patients ( 1 (3\%) ST patient, $5(12 \%)$ TM patients) and wound infection/healing problems in 7 patients (4 (12\%) ST patients, 3 (7\%) TM patients). A transurethral catheter was placed postoperatively in $6(19 \%)$ ST and in 7 (16\%) TM patients and a pressure bandage in all patients. The pressure bandage remained for an average of 2.3 days $(1-4)$ in ST and 2.9 days (1-6) in TM patients $(P<0.05)$. The mean time of hospitalisation was 6.6 days (4-23) in the ST and 6.3 days $(4-15)$ in the TM group $(P=0.7)$. 
Table 1 Synopsis of pre- and perioperative data in all patients either operated by STs or the TM.

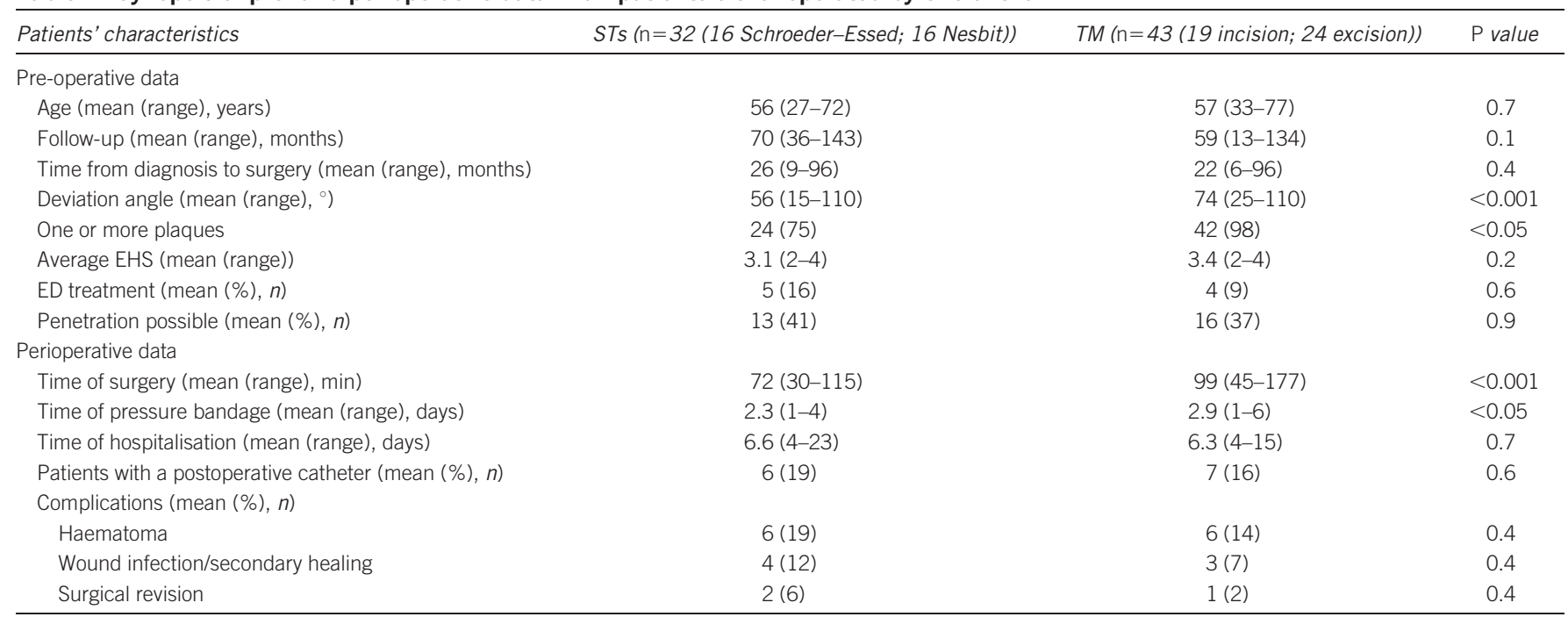

Abbreviations: ED, erectile dysfunction; EHS, erection hardness score; ST, shortening technique; TM, TachoSil method. $P$ values of $<0.05$ were considered significant.

\section{RESULTS}

Results of the self-reported questionnaires

Self-reported postoperative complications did not show significant differences between both groups. With regard to penile straightening $21(66 \%)$ ST patients and $36(84 \%)$ TM patients reported that they currently had a straight or almost straight penis. Ten (31\%) ST and 4 (9\%) TM patients stated that their penis was straighter than before and in each group $1(3 \%)$ ST and $3(7 \%)$ TM patients stated that results were worse than before. Even if results of the TM group were slightly better with regard to the higher preoperative deviation, differences were not significant $(P=0.2)$ (Figure 1).

Regarding erectile function, ST patients currently reported an average EHS of 3.3 (EHS 1: $n=0$; EHS 2: $n=5$; EHS 3: $n=12$; EHS 4: $n=15$ ) and TM patients a score of 2.6 (EHS 1: $n=3$; EHS 2: $n=21$; EHS 3: $n=8$; EHS 4: $n=11$ ) (Figure 2; Table 2). Differences were statistically significant between both groups $(P<0.001)$ (Figure 2; Table 2). Three months postoperatively, ST patients reported an average EHS of 3.2 (EHS 1: $n=0$; EHS 2: $n=7$; EHS $3: n=11$; EHS $4: n=14$ ) and TM

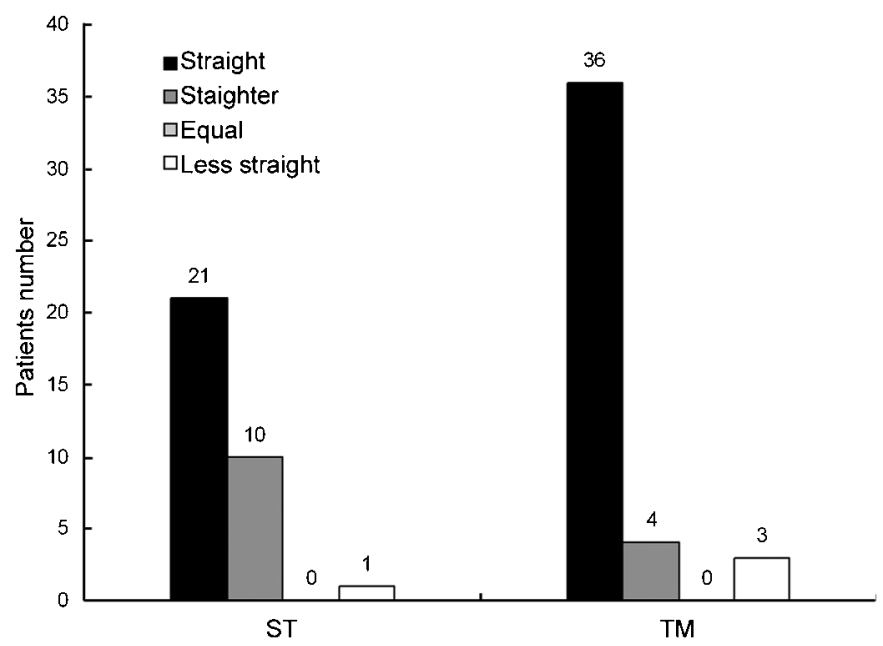

Figure 1 Self-reported current straightening results of both groups (ST and TM). Differences between both groups were not significant $(P=0.2)$. ST, shortening technique; TM, TachoSil method. patients of 2.5 (EHS 1: $n=6$; EHS 2: $n=17$; EHS 3: $n=11$; EHS 4: $n=9) \quad(P<0.001$; Table 2). Nevertheless, only $3(9 \%)$ ST and 9 (21\%) TM patients stated that they were actually under evidencebased treatment including phosphodiesterase 5 inhibitors, alprostadil or vacuum devices (Table 2 ).

Penile shortening was self-reported by 23 (72\%) ST and 40 (93\%) TM patients $(P<0.05$; Table 2). One (3\%) ST and 7 (16\%) TM patients reported a severe loss of sensation $(P=0.08)$. Moderate loss of sensation was described in 13 (41\%) ST and 18 (42\%) TM patients. Five (16\%) ST and $11(26 \%)$ TM patients currently reported orgasmic disorders. Absence of pain was currently reported by 31 (97\%) ST and $40(93 \%)$ TM patients in the case of a flaccid penis and by 27 (84\%) ST and 38 (88\%) TM during erections.

Regarding satisfaction, 20 (62\%) ST and 22 (51\%) TM patients reported that currently sexual intercourse was satisfactory to them in at least $50 \%$ of the cases ( $P=0.4$; Table 2$)$. Fourteen (44\%) ST versus 19 (44\%) TM patients described that their situation had improved,

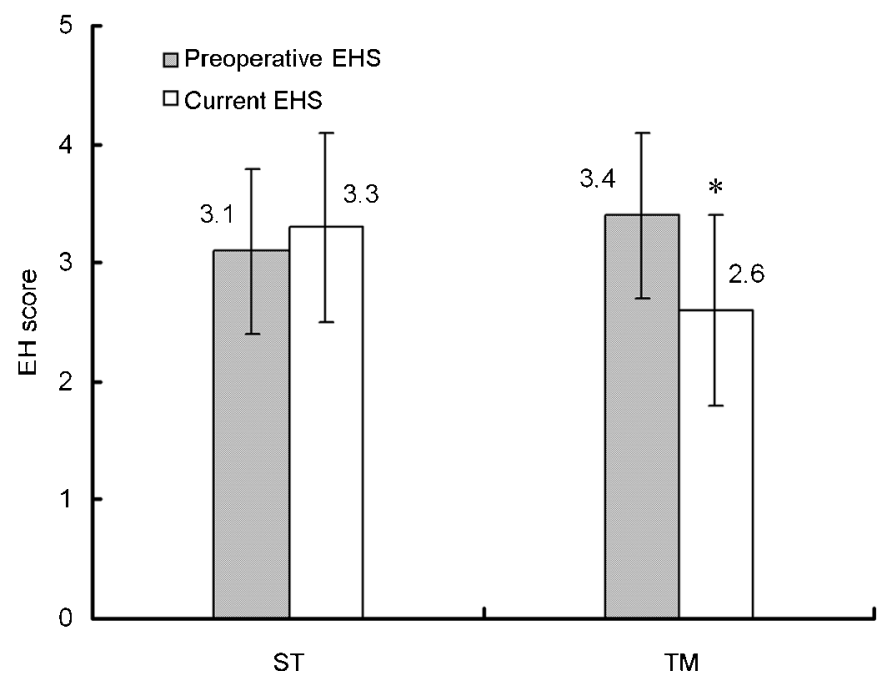

Figure 2 Mean preoperative versus current self-reported EHS. $* P<0.001$, compared with current EHS in ST group. EHS, erection hardness score; ST, shortening technique; TM, TachoSil method. 
Table 2 Self-reported follow-up data in all patients operated by either STs or the TM.

\begin{tabular}{|c|c|c|c|}
\hline Self-reported follow-up data & STs $(\mathrm{n}=32(16$ Schroeder-Essed; 16 Nesbit $))$ & $T M(n=43$ (19 incision; 24 excision $))$ & $\mathrm{P}$ value \\
\hline \multicolumn{4}{|l|}{ Penis: straight/straighter than before/equal/less straight $(n)$} \\
\hline 3 months postoperative & 25/6/0/1 & $41 / 1 / 0 / 1$ & 0.8 \\
\hline Current situation & 21/10/0/1 & $36 / 4 / 0 / 3$ & 0.2 \\
\hline Penile shortening $(n(\%))$ & $23(72)$ & $40(93)$ & $<0.05$ \\
\hline Loss of sensation (severe/moderate) & $1 / 13$ & $7 / 16$ & 0.08 \\
\hline \multicolumn{4}{|l|}{ Average EHS (mean (range)) } \\
\hline 3 months postoperative & $3.2(2-4)$ & $2.5(1-4)$ & $<0.001$ \\
\hline Current situation & $3.3(2-4)$ & $2.6(1-4)$ & $<0.001$ \\
\hline ED treatment $(n(\%))$ & $3(9)$ & $9(21)$ & 0.2 \\
\hline \multicolumn{4}{|l|}{ Satisfaction } \\
\hline Satisfactory sexual intercourse in $>50 \%$ of the cases $(n(\%))$ & $20(62)$ & $22(51)$ & 0.4 \\
\hline Situation: improved/equal/worse $(n)$ & $14 / 8 / 10$ & $19 / 7 / 17$ & 0.6 \\
\hline Satisfaction: satisfied/neutral/dissatisfied ( $n$ ) & $13 / 6 / 13$ & 20/6/17 & 0.9 \\
\hline Would you again choose the same intervention: yes versus no $(n(\%))$ & $19(59)$ versus $13(41)$ & $24(56)$ versus $19(44)$ & 0.8 \\
\hline
\end{tabular}

Abbreviations: ED, erectile dysfunction; EHS, erection hardness score; ST, shortening technique; TM, TachoSil method. $P$ values of $<0.05$ were considered significant.

whereas $10(31 \%)$ ST and $17(40 \%)$ TM patients stated that their situation became worse. Thirteen (41\%) ST and 20 (47\%) TM patients described that they were satisfied with the current situation whereas 13 (41\%) ST and 17 (40\%) TM patients described that they were not. In each group, 6 patients remained undecided regarding this question. Nineteen (59\%) ST and 24 (56\%) TM patients stated that they retrospectively would choose the same surgical intervention. Satisfaction rates were similar in both groups. No statistical differences were found (Table 2).

\section{DISCUSSION}

$\mathrm{PD}$ is a bothersome disease that strongly affects quality of life. Conservative treatments are often of limited success and many men seek help in surgical correction during the course of their disease. Different surgical techniques have been described mainly including STs or lengthening procedures with plaque incision versus excision and defect covering. ${ }^{19,33}$

To enable recommendation of the best surgical approach, there is still a need for long-term evaluations especially with regard to quality of life. ${ }^{13,19,34}$ In the present study, we evaluated pre- and perioperative data and self-reported long-term results of patients who underwent surgery for penile correction either by shortening techniques (ST group) or a grafting procedure with TachoSil (TM group). Both groups were similar regarding age, comorbidities, preoperative treatment and erectile function, except for a higher preoperative deviation angle and a higher incidence of plaques in TM group patients.

Both surgical techniques (ST and TM) were shown to be safe without significant differences in complication rates. In long term regarding penile straightening the grafting procedure (TM) appeared to be more successful than the ST procedures considering the fact that preoperative penile deformities were even higher in TM than in ST patients. These results provide evidence for a successful surgical treatment of penile deformity in PD using both techniques dependent to individual decisions. In literature of long-term follow-up successful straightening results were reported for both ST and grafting techniques with similar outcome results as shown in our study. ${ }^{21,27}$

Regarding erectile function ST patients self-reported significantly better results than TM patients during the follow-up. In ST patients, the average preoperative EHS did not change postoperatively (average EHS pre-operatively $3.2,3.2$ at 3 months postoperatively and 3.3 currently). In contrast to that a significant loss of erectile function was noted for patients after the grafting procedure (average EHS pre-operatively $3.4,3$ months postoperatively 2.4 and currently 2.6; $P<0.05)$. According to the authors and others, ${ }^{19}$ these results must be attributed to the more severe surgical trauma consisting of a significantly longer time of surgery, mobilisation of the neurovascular bundle, and opening of the tunica albuginea during the TM procedures. Results of erectile function in the present study parallel those of other self-reported long-term studies. Even though some studies described excellent results after grafting procedures, ${ }^{26,35}$ others reported, in accordance with our observations, a reduced erectile function of patients after a grafting procedure. ${ }^{21,23,27} \mathrm{Hsu}$ et al.$^{26}$ reported equivalent erectile function after venous grafting procedures in comparison to the preoperative situation in patients with $\mathrm{PD}$ and with congenital deviation after an average follow-up of 43 months ( 7 month to 10 years), whereas Kim et al. ${ }^{27}$ described a significantly reduced erectile function/rigidity after a grafting procedure with venous patches after a medium follow-up time of at least 1 year compared to simpler shortening procedures. Also in line with the results of our study, Kim et al. ${ }^{27}$ described a higher loss of sensation in patients after grafting procedure than after ST procedures even though differences in our study were statistically not significant. In addition, in our study TM patients—-resulting in slightly increased paresthesia-more often presented with dorsal plaques making a mobilisation of the neurovascular bundle with possible nerve damage necessary. Patients of both groups described penile shortening ranging from one to a few centimetres. This was also described by Kim et al. ${ }^{27}$ who found self-reported penile shortening in patients after venous grafting and STs in a longterm follow-up. Interestingly, patients of this study even reported significantly worse results in the TM group in comparison to the ST group $(P<0.05)$. This might be at least partially attributed to the reduced erectile function in this group of patients and the fact of subjective evaluation, but is of course in contrast to the common belief that lengthening of the concave curved side of the penis as done in grafting procedures avoids penile shortening.

Probably due to a higher relief of patients in the TM group because of the more severe penile deformity and finally a better erectile function in the ST group, overall patient's satisfaction rates were similar in both groups, even though at a relatively low level. These results are similar with those of other studies in which patients reported also low satisfaction rates after a long-term follow-up. ${ }^{21,23,27}$ The relatively low satisfaction rates in the present study and other 
questionnaire-based studies also need to be judged under the perspective that such questionnaire-based studies tend to lead to significantly less positive results than direct interviews as reported by van der Horst et al. ${ }^{34}$

As a limitation of the present study data acquisition has to be discussed. All pre- and perioperative data were obtained retrospectively by chart review, and even though a complete medical history was available for all patients, this approach could have had an influence on the kind of data selected. All 3-month interim and current patient's data were obtained subjectively by questionnaires even though some of them, for example penile length or penile straightening, might have been objectively assessed by simple measurements of preoperative versus postoperative length or deviation angle which was not evaluated in our study. However, it was our intention to emphasize on the patient subjective satisfaction rates in this study. Highly subjective patient's perceptions in erectile function, and penile sensation contributing to satisfaction rates can, to our believe and others, ${ }^{36}$ best be evaluated by asking the patients directly, as was done in our questionnaire.

Finally, the present study cannot serve for a comparison of the TM to other different grafting materials described in the literature, although this would be of high interest, since there is a paucity of direct tissue proof. This is especially true as TachoSil seems to be in comparison to other grafting materials the ideal graft for corporotomy defects because of its easy application and the fact that it might serve as an ideal matrix for the spontaneous healing of the tunica albuginea. Still no such studies have been reported yet.

As a conclusion from the present study we found, that both the ST and TM revealed to be safe and sustainable successful in penile straightening with similar patient satisfaction rates. However, in comparison to patients operated according to the TM, ST patients revealed a better self-reported functional outcome regarding erectile function, penile length and sensation.

\section{AUTHOR CONTRIBUTIONS}

$\mathrm{MH}$ was responsible for the conceptual design of the study, developed the questionnaire, analysed data and wrote the paper; MK developed the questionnaire and was together with $\mathrm{JH}$ responsible for data acquisition and statistical analysis, BA and AS substantially contributed to the interpretation of the data and revised the final manuscript.

\section{COMPETING FINANCIAL INTERESTS}

The authors declare no competing financial interests.

1 Devine CJ Jr, Somers KD, Ladaga LE. Peyronie's disease: pathophysiology. Prog Clin Bioles 1991; 370: 355-8.

2 Gonzalez-Cadavid NF. Mechanisms of penile fibrosis. J Sex Med 2009; Suppl 3, 353-62.

3 Sommer F, Schwarzer U, Wassmer G, Bloch W, Braun M et al. Epidemiology of Peyronie's disease. Int J Impot Res 2002; 14: 379-83.

4 Schwarzer U, Sommer F, Klotz T, Braun M, Reifenrath B et al. The prevalence of Peyronie's disease: results of a large survey. BJU Int 2001; 88: 727-30.

5 Sasso F, Gulino G, Falabella R, D'Addessi A, Sacco E et al. Peyronie's disease: lights and shadows. Urol Int 2007; 78: 1-9.

6 Rhoden EL, Riedner CE, Fuchs S, Ribeiro EP, Halmenschlager G. A cross-sectional study for the analysis of clinical, sexual and laboratory conditions associated to Peyronie's disease. J Sex Med 2010; 7: 1529-37.

7 Hauck EW, Diemer T, Weidner W. Peyronie's disease. Conservative and surgical therapy. Urologe A 2006; 45: W243-57. German.

8 Gonzalez-Cadavid NF, Rajfer J. Mechanisms of disease: new insights into the cellular and molecular pathology of Peyronie's disease. Nat Clin Pract Urol 2005; 2: 291-7.

9 Trost LW, Gur S, Hellstrom WJ. Pharmacological management of Peyronie's disease. Drugs 2007; 67: 527-45

10 Soh J, Kawauchi A, Kanemitsu N, Naya Y, Ochiai A et al. Nicardipine vs. saline injection as treatment for Peyronie's disease: a prospective, randomized, singleblind trial. J Sex Med 2010; 7: 3743-9.
11 Palmieri A, Imbimbo C, Longo N, Fusco F, Verze et al. A first prospective, randomized, double-blind, placebo-controlled clinical trial evaluating extracorporeal shock wave therapy for the treatment of Peyronie's disease. Eur Urol 2009; 56: 363-9.

12 Chitale S, Morsey M, Swift L, Sethia K. Limited shock wave therapy vs sham treatment in men with Peyronie's disease: results of a prospective randomized controlled doubleblind trial. BJU Int 2010; 106: 1352-6.

13 Ralph D, Gonzalez-Cadavid N, Mirone V, Perovic S, Sohn M et al. The management of Peyronie's disease: evidence-based 2010 guidelines. J Sex Med 2010; 7: 2359-74.

14 Kadioglu A, Tefekli A, Erol B, Oktar T, Tunc M et al. A retrospective review of 307 men with Peyronie's disease. J Urol 2002; 168: 1075-9.

15 Essed E, Schroeder FH. New surgical treatment for Peyronie disease. Urology 1985; 25: 582-7.

16 Nesbit RM. Congenital curvature of the phallus: report of three cases with description of corrective operation. J Urol 1965; 93: 230-2.

17 Levine LA, Lenting EL. A surgical algorithm for the treatment of Peyronie's disease. J Urol 1997; 158: 2149-52.

18 Lentz AC, Carson CC 3rd. Peyronie's surgery: graft choices and outcomes. Curr Urol Rep 2009; 10: 460-7.

19 Tran VQ, Kim DH, Lesser TF, Aboseif SR. Review of the surgical approaches for Peyronie's disease: corporeal plication and plaque incision with grafting. Adv Urol 2008: 263450

20 Lue TF, El-Sakka Al. Venous patch graft for Peyronie's disease. Part I: technique. J Urol 1998; 160: 2047-9.

21 Taylor FL, Levine LA. Surgical correction of Peyronie's disease via tunica albuginea plication or partial plaque excision with pericardial graft: long-term follow up. J Sex Med 2008; 5: 2221-8; discussion 2229-30.

22 Austoni E, Fenice O, Kartalas-Goumas Y, Colombo F, Mantovani F et al Radical surgical treatment of Peironie disease by excision of the plaque and dermal graft allowing conservation of erection. Ann Urol (Paris) 1996; 30: 204-12. French.

23 Simonato A, Gregori A, Varca V, Venzano F, de Rose AF et al. Penile dermal flap in patients with Peyronie's disease: long-term results. J Urol 2010; 183: 1065-8.

24 Gulino G, Falabella R, Gentile G, Sasso F. Radical surgery in Peyronie's disease. Graft comparison. Minerva Chir 2002; 57: 383-8.

25 Lahme S, Gotz T, Bichler KH. Collagen fleece for defect coverage following plaque excision in patients with Peyronie's disease. Eur Urol 2002; 41: 401-5.

26 Hsu GL, Chen HS, Hsieh CH, Chen RM, Wen HS et al. Long-term results of autologous venous grafts for penile morphological reconstruction. J Androl 2007; 28: 186-93.

$27 \mathrm{Kim} \mathrm{DH}$, Lesser TF, Aboseif SR. Subjective patient-reported experiences after surgery for Peyronie's disease: corporeal plication versus plaque incision with vein graft. Urology 2008; 71: 698-702.

28 Mulhall JP, Goldstein I, Bushmakin AG, Cappelleri JC, Hvidsten K. Validation of the erection hardness score. J Sex Med 2007; 4: 1626-34.

29 Goldstein I, Mulhall JP, Bushmakin AG, Cappelleri JC, Hvidsten K et al. The erection hardness score and its relationship to successful sexual intercourse. J Sex Med2008; 5: 2374-80.

30 Frilling A, Stavrou GA, Mischinger HJ, de Hemptinne B, Rokkjaer M et al. Effectiveness of a new carrier-bound fibrin sealant versus argon beamer as haemostatic agent during liver resection: a randomised prospective trial. Langenbecks Arch Surg 2005; 390: 114-20.

31 Anegg U, Lindenmann J, Matzi V, Smolle J, Maier A et al. Efficiency of fleece-bound sealing (TachoSil) of air leaks in lung surgery: a prospective randomised trial. Eur J Cardiothorac Surg 2007; 31: 198-202.

32 Siemer S, Lahme S, Altziebler S, Machtens S, Strohmaier W et al. Efficacy and safety of TachoSil as haemostatic treatment versus standard suturing in kidney tumour resection: a randomised prospective study. Eur Urol 2007; 52: 1156-63.

33 Egydio PH. Surgical treatment of Peyronie's disease: choosing the best approach to improve patient satisfaction. Asian J Androl 2008; 10: 158-66.

34 van der Horst C, Martinez Portillo FJ, Seif C, Alken P, Juenemann KP. Treatment of penile curvature with Essed-Schroder tunical plication: aspects of quality of life from the patients' perspective. BJU Int 2004; 93: 105-8.

$35 \mathrm{Hsu}$ GL. Hypothesis of human penile anatomy, erection hemodynamics and their clinical applications. Asian J Androl 2006; 8: 225-34.

36 Friedrich MG, Evans D, Noldus J, Huland $H$. The correction of penile curvature with the Essed-Schroder technique: a long-term follow-up assessing functional aspects and quality of life. BJU Int 2000; 86: 1034-8.

\section{APPENDIX}

Please mark the appropriate answer

1. Which problems occurred directly after the operation? (Several answers are possible)

a) Wound infection

b) Voiding became impossible.

c) Voiding was possible only with major difficulties.

d) Haematoma

e) A second intervention became necessary (within 3 months).

f) Strong pain

g) Other complications 
2. Was your penis straight 3 months after the operation?
a) It was straight.
b) It was almost straight.
c) It was a little straighter than before.
d) It was as curved as before.
e) It was more curved than before.

3. Is your penis straight today?
a) It is straight.
b) It is almost straight.
c) It is almost as curved as before the operation.
d) It is as curved as before.
e) It is more curved than before.

4. Did your penis become shorter?
a) It is not shorter than before the operation.
b) It is about $0-1 \mathrm{~cm}$ shorter.
c) It is about $1-2 \mathrm{~cm}$ shorter.
d) It is more than $2 \mathrm{~cm}$ shorter.

5. Do you suffer from sensory loss in the penis?
a) No
b) Yes, it is less sensitive.
c) Yes, I only remark strong pain stimulus.
d) Yes, it is completely insensitive.

6. How is your maximal erectile capacity today?

a) The penis becomes completely hard and fully rigid.

b) The penis is hard enough for penetration, but not completely hard.

c) The penis is hard, but not hard enough for penetration.

d) The penis becomes larger, but not hard.

7. How was your maximal erectile capacity 3 months after the operation?

a) The penis becomes completely hard and fully rigid.

b) The penis is hard enough for penetration, but not completely hard.

c) The penis is hard, but not hard enough for penetration.

d) The penis becomes larger, but not hard.

8. How often was sexual intercourse satisfactory to you after the operation?
a) Almost always
b) Most of the times (more often than half of the cases)
c) Sometimes (in about half of the cases)
d) Seldom (less often than half of the cases)
e) Almost never

9. Do you suffer from ejaculatory or orgasmic problems?
a) Never
b) Seldom (less often than in half of the cases)
c) Sometimes (in about half of the cases)
d) Most of the times (more often than half of the cases)
e) Almost always

10. Do you feel pain in the penis in a flaccid situation?
a) No, it does not hurt.
b) Yes, it hurts a little, but it does not disturb me.
c) Yes, it hurts and it disturbs me.
d) Yes, it hurts a lot. Because of that normal life is not possible.

11. Do you suffer from pain during erection?
a) No, it does not hurt.
b) Yes, it hurts but does not disturb sexual intercourse.
c) Yes, it hurts and disturbs sexual intercourse.
d) Yes, it hurts a lot. Because of that sexual intercourse is impossible.

12. Do you use any treatment to achieve better erections?
a) PDE-5 inhibitors (Viagra $\AA$, Cialis $\AA$, Levitra $\AA$ )
b) Application of Alprostadil into the urethra (MUSE®)
c) Injection of Alprostadil into the corpora cavernosa (SKAT)
d) Vacuum devices

13. Did your situation improve because of the operation?
a) It improved a lot.
b) It improved a little.
c) It neither improved nor became worse.
d) It became a little bit worse.
e) It became much worse.

14. Have you been satisfied with the results of the operation?
a) Very satisfied
b) Satisfied
c) Neither satisfied nor dissatisfied
d) Dissatisfied
e) Completely dissatisfied

15. Would you again choose this surgical treatment?
a) Yes
b) No

16. Have you been operated again on the penis afterwards?
a) $\mathrm{No}$
b) Yes If yes, then why 